\title{
Certain Generating Relations Involving the Generalized Multi-Index Bessel-Maitland Function
}

\author{
Shilpi Jain, ${ }^{1}$ Juan J. Nieto ${ }^{D},{ }^{2}$ Gurmej Singh, ${ }^{3,4}$ and Junesang Choi ${ }^{5}$ \\ ${ }^{1}$ Department of Mathematics, Poornima College of Engineering, Jaipur 302022, India \\ ${ }^{2}$ Instituto de Matematicas, Universidade de Santiago de Compostela, 15782 Santiago de Compostela, Spain \\ ${ }^{3}$ Department of Mathematics, Mata Sahib Kaur Girls College, Talwandi Sabo, Bathinda 151103, India \\ ${ }^{4}$ Department of Mathematics, Singhania University, Pacheri Bari, Jhunjhunu, India \\ ${ }^{5}$ Department of Mathematics, Dongguk University, Gyeongju 38066, Republic of Korea
}

Correspondence should be addressed to Juan J. Nieto; juanjose.nieto.roig@usc.es

Received 29 April 2020; Accepted 4 August 2020; Published 25 August 2020

Academic Editor: Luigi Rodino

Copyright (c) 2020 Shilpi Jain et al. This is an open access article distributed under the Creative Commons Attribution License, which permits unrestricted use, distribution, and reproduction in any medium, provided the original work is properly cited.

Generating relations involving the special functions have already proved their important role in mathematics and other fields of sciences. In this paper, we aim to provide some presumably new generating relations in connection with the generalized multiindex Bessel-Maitland function $J_{\left(v_{j_{m}}, q\right.}^{\left(\left(_{j}, q\right.\right.}$ (.). The main results presented here, being very general, can yield a number of particular or equivalent identities, some of which are explicitly demonstrated.

\section{Introduction and Preliminaries}

Here and elsewhere, let $\mathbb{C}, \mathbb{R}, \mathbb{R}^{+}, \mathbb{N}$, and $\mathbb{Z}_{0}^{-}$be the sets of complex numbers, real numbers, positive real numbers, positive integers, and nonpositive integers, respectively.

The Bessel-Maitland function $J_{\nu}^{\lambda}(z)$ is defined as (see Marichev [1])

$$
J_{\nu}^{\lambda}(z)=\sum_{r=0}^{\infty} \frac{(-z)^{r}}{\Gamma(\lambda r+\nu+1) r !}, \quad \lambda \in \mathbb{R}^{+}, z \in \mathbb{C} .
$$

Pathak [2] gave the following more generalized form of generalized Bessel-Maitland function (1):

$$
\begin{gathered}
J_{\nu, q}^{\lambda, \gamma}(z)=\sum_{r=0}^{\infty} \frac{(\gamma)_{q r}}{\Gamma(\lambda r+\nu+1)} \frac{(-z)^{r}}{r !} \\
(\lambda, \nu, \gamma \in \mathbb{C}, \mathfrak{R}(\lambda) \geq 0, \mathfrak{R}(\nu) \geq-1, \mathfrak{R}(\gamma) \geq 0, q \in(0,1) \cup \mathbb{N}) .
\end{gathered}
$$

Remark 1. Even though Pathak excluded $q=0$ in (2), the case $q=0$ yields (1).
If $q=1, \gamma=1, v$ is replaced by $v-1$, and $z$ is replaced by $-z$ in (2), then generalized Bessel-Maitland function reduces to the Mittag-Leffler function which was studied by Wiman [3] as follows:

$$
J_{\nu-1,1}^{\lambda, 1}(-z)=E_{\lambda, \nu}(z), \quad \mathfrak{R}(\lambda)>0, \mathfrak{R}(\nu)>0 .
$$

If $v$ is replaced by $v-1$ and $z$ is replaced by $-z$ in (2), then the generalized Bessel-Maitland function reduces to the well-known generalized Mittag-Leffler function $E_{\lambda, \nu}^{\gamma, q}(z)$ which was introduced by Shukla and Prajapati [4] as follows:

$$
J_{\nu-1, q}^{\lambda, \gamma}(-z)=E_{\lambda, \nu}^{\gamma, q}(z)
$$

$$
(\Re(\lambda)>0, \mathfrak{R}(\nu)>0, \mathfrak{R}(\gamma)>0 ; q \in(0,1) \cup \mathbb{N}) .
$$

Jain and Agarwal [5] generalized Bessel-Maitland function $J_{v}^{\lambda}(z)(1)$ as follows:

$$
J_{\nu, \mu}^{\lambda}(z)=\sum_{r=0}^{\infty} \frac{(-1)^{r}(z / 2)^{\nu+2 \mu+2 r}}{\Gamma(\lambda r+v+\mu+1) \Gamma(\mu+r+1)},
$$

$$
\left(\lambda \in \mathbb{R}^{+}, \nu, \mu \in \mathbb{C}, z \in \mathbb{C} \backslash(-\infty, 0]\right) .
$$


Choi and Agarwal [6] investigated the following generalized multi-index Bessel function:

$$
J \frac{\left(\lambda_{j}\right)_{m, \gamma}}{\left(v_{j}\right)_{m, q}}(z)=\sum_{r=0}^{\infty} \frac{(\gamma)_{q r}}{\prod_{j=1}^{m} \Gamma\left(\lambda_{j} r+v_{j}+1\right)} \frac{(-z)^{r}}{r !}
$$

where $m \in \mathbb{N}$ and $\lambda_{j}, v_{j}, \gamma, q$, and $z \in \mathbb{C}(j=1, \ldots, m)$ such that

$$
\sum_{j=1}^{m} \mathfrak{R}\left(\lambda_{j}\right)>\max \{0, \mathfrak{R}(q)-1\}, \quad \mathfrak{R}\left(\nu_{j}\right)>-1, \mathfrak{R}(\gamma)>0, q \in(0,1) \cup \mathbb{N}
$$

Remark 2. It is easily found that generalized multi-index Bessel-Maitland function (9) is equivalent to the generalized multi-index Mittag-Leffler function defined and studied by Saxena and Nishimoto [7] (see also [8]).

Pohlen [9] introduced the Hadamard product (or the convolution) $f * g$ of two analytic functions $f$ and $g$ as follows:

$$
(f * g)(z):=\sum_{n=0}^{\infty} a_{n} b_{n} z^{n}=(g * f)(z), \quad(|z|<R),
$$

where $R \geq R_{f} \cdot R_{g}$. Here, $f(z)$ and $g(z)$ are analytic at $z=0$ whose Maclaurin series with their respective radii of convergence $R_{f}$ and $R_{g}$ are

$$
\begin{array}{ll}
f(z)=\sum_{n=0}^{\infty} a_{n} z^{n}, & \left(|z|<R_{f}\right), \\
g(z)=\sum_{n=0}^{\infty} b_{n} z^{n}, & \left(|z|<R_{g}\right) .
\end{array}
$$

The concept of the Hadamard product has turned out to be useful, particularly, in factorizing a newborn function, which is usually expressed as a Maclaurin series, into two known functions (see, e.g., [10-13]).

The $k$-th derivative of the function $f(p)=p^{-\lambda-n \xi}(\lambda, \xi \in \mathbb{C}, n \in \mathbb{N})$ is easily found to be given in terms of gamma function as follows:

$$
f^{(k)}(p)=(-1)^{k} p^{-\lambda-n \xi-k} \frac{\Gamma(\lambda+n \xi+k)}{\Gamma(\lambda+n \xi)}, \quad\left(k \in \mathbb{N}_{0}\right)
$$

Generating functions have been widely used in exploring certain properties and formulas involving sequences and polynomials in a wide range of research subjects. Many researchers have developed a remarkably large number of generating functions associated with a variety of special functions. For some works on this subject, one may refer, for example, to an extensive monograph [14-25] and the literature cited therein. In this search, we aim to provide some presumably new generating relations in connection with generalized multi-index Bessel-Maitland function (9). The main results developed here, being very general, can be reduced to produce a large number of presumably new and potentially useful generating relations for other known functions, some of which are demonstrated.

\section{Generating Relations}

We give two generating relations involving generalized multi-index Bessel-Maitland function (9) asserted by the following theorems.

Theorem 1. Let $m \in \mathbb{N}$ and $\lambda_{j}, v_{j}, \gamma, q$, and $z \in \mathbb{C}(j=$ $1, \ldots, m)$ such that

$$
\sum_{j=1}^{m} \mathfrak{R}\left(\lambda_{j}\right)>\max \{0, \mathfrak{R}(q)-1\}, \quad \mathfrak{R}\left(v_{j}\right)>-1, \mathfrak{R}(\gamma)>0, q \in(0,1) \cup \mathbb{N} .
$$

Also, let $|t|<1$. Then,

$$
\begin{aligned}
& (1+t)^{-\sigma} J \begin{array}{l}
\left(\lambda_{j}\right)_{m}, \gamma \\
\left(v_{j}\right)_{m}, q
\end{array}\left(\frac{z}{1+t}\right) \\
& \quad=\sum_{k=0}^{\infty}(-1)^{k}(\sigma)_{k} J \frac{\left(\lambda_{j}\right)_{m}, \gamma}{\left(v_{j}\right)_{m}, q}(z) *{ }_{1} F_{1}(\sigma+k ; \sigma ;-z) \frac{t^{k}}{k !} .
\end{aligned}
$$

Proof. We replace $1+t$ by $s$ in the left-hand side of (15) and denote the resulting expression by $g(s)$. Then, using form (9), on expanding the function in series, gives

$$
g(s)=s^{-\sigma} J \frac{\left(\lambda_{j}\right)_{m, \gamma}}{\left(v_{j}\right)_{m, q}}\left(\frac{z}{s}\right)=\sum_{r=0}^{\infty} \frac{(\gamma)_{q r}}{\prod_{j=1}^{m} \Gamma\left(\lambda_{j} r+v_{j}+1\right)} \frac{(-z)^{r}}{r !} s^{-\sigma-r} .
$$

Differentiating $k$ times both sides of (16) with respect to $s$ with the aid of (13) (term-by-term differentiation can be verified under the given conditions), we find

$$
g^{(k)}(s)=(-1)^{k} s^{-\sigma-k} \sum_{r=0}^{\infty} \frac{(\gamma)_{q r}}{\prod_{j=1}^{m} \Gamma\left(\lambda_{j} r+v_{j}+1\right)} \frac{\Gamma(\sigma+r+k)}{\Gamma(\sigma+r)}\left(\frac{-z}{s}\right)^{r} \frac{1}{r !},
$$


which is simplified to yield

$$
g^{(k)}(s)=(-1)^{k} s^{-\sigma-k}(\sigma)_{k} \sum_{r=0}^{\infty} \frac{(\gamma)_{q r}}{\prod_{j=1}^{m} \Gamma\left(\lambda_{j} r+\nu_{j}+1\right)} \frac{(\sigma+k)_{r}}{(\sigma)_{r}}\left(\frac{-z}{s}\right)^{r} \frac{1}{r !} .
$$

Decomposing series (18) into Hadamard product (11), we obtain

$$
g^{(k)}(s)=(-1)^{k} s^{-\sigma-k}(\sigma)_{k} J \frac{\left(\lambda_{j}\right)_{m} \gamma}{\left(v_{j}\right)_{m}, q}\left(\frac{z}{s}\right) *{ }_{1} F_{1}\left(\sigma+k ; \sigma ;-\frac{z}{s}\right) .
$$

Expanding $g(s+t)$ as the Taylor series gives

$$
g(s+t)=\sum_{k=0}^{\infty} \frac{t^{k}}{k !} g^{(k)}(s) .
$$

Combining (16), (19), and (20), we obtain

$$
\begin{aligned}
& (s+t)^{-\sigma} J \frac{\left(\lambda_{j}\right)_{m}, \gamma}{\left(v_{j}\right)_{m}, q}\left(\frac{z}{s+t}\right)=\sum_{k=0}^{\infty} \frac{(-t)^{k} s^{-\sigma-k}}{k !}(\sigma)_{k} J \begin{array}{l}
\left(\lambda_{j}\right)_{m}, \gamma \\
\left(v_{j}\right)_{m}, q
\end{array}\left(\frac{z}{s}\right) \\
& *{ }_{1} F_{1}\left(\sigma+k ; \sigma ;-\frac{z}{s}\right) .
\end{aligned}
$$

Finally, setting $s=1$ yields desired result (15).

Theorem 2. Let $m \in \mathbb{N}$ and $\lambda_{j}, v_{j}, \gamma, q$, and $z \in \mathbb{C}(j=$ $1, \ldots, m)$ such that

$\sum_{j=1}^{m} \mathfrak{R}\left(\lambda_{j}\right)>\max \{0, \mathfrak{R}(q)-1\}, \quad \mathfrak{R}\left(v_{j}\right)>-1, \mathfrak{R}(\gamma)>0, q \in(0,1) \cup \mathbb{N}$.

Also, let $|t|<1$. Then,

$$
\left.\sum_{k=0}^{\infty}\left(\begin{array}{c}
\gamma+k-1 \\
k
\end{array}\right) J \begin{array}{l}
\left(\lambda_{j}\right)_{m}, \gamma+k \\
\left(\nu_{j}\right)_{m}, q
\end{array}(z) t^{k}=(1-t)^{-\gamma} J \begin{array}{l}
\left(\lambda_{j}\right)_{m, \gamma},\left(v_{j}\right)_{m}, q \\
(1-t)^{q}
\end{array}\right) .
$$

Proof. Let J be the left-hand side of (23). Using (9), on expanding the function in series, gives

$$
\mathrm{J}=\sum_{k=0}^{\infty}\left(\begin{array}{c}
\gamma+k-1 \\
k
\end{array}\right)\left\{\sum_{r=0}^{\infty} \frac{(\gamma+k)_{q r}}{\prod_{j=1}^{m} \Gamma\left(\lambda_{j} r+v_{j}+1\right)} \frac{(-z)^{r}}{r !}\right\} t^{k} .
$$

Interchanging the order of summations in (24) and using the known identity (see, e.g., [26, p. 5])

$$
\left(\begin{array}{l}
\gamma \\
k
\end{array}\right)=\frac{\Gamma(\gamma+1)}{k ! \Gamma(\gamma-k+1)}, \quad k \in \mathbb{N}_{0}, \gamma \in \mathbb{C}
$$

we have
$\mathrm{J}=\sum_{r=0}^{\infty} \frac{(\gamma)_{q r}}{\prod_{j=1}^{m} \Gamma\left(\lambda_{j} r+v_{j}+1\right)}\left\{\sum_{k=0}^{\infty}\left(\begin{array}{c}\gamma+q r+k-1 \\ k\end{array}\right) t^{k}\right\} \frac{(-z)^{r}}{r !}$.

Using the generalized binomial expansion, we find that the inner sum in (26) gives

$$
\sum_{k=0}^{\infty}\left(\begin{array}{c}
\gamma+q r+k-1 \\
k
\end{array}\right) t^{k}=(1-t)^{-(\gamma+q r)}, \quad|t|<1 .
$$

Finally, interpreting (26) with the help of (27) yields desired result (23).

\section{Further Remarks}

Here, we choose to give some equivalent identities and particular cases of the results in Theorems 1 and 2. As noted in Remark 2 , setting $v_{j}$ by $v_{j}-1$ and $z$ by $-z$ in (15) and (23) gives two corresponding generating relations involving the generalized multi-index Mittag-Leffler function $E_{\left(\lambda, \nu_{j}\right)_{m}}^{\gamma, q}(z)$, which are asserted, respectively, in Corollaries 1 and 2 .

Corollary 1. Let $m \in \mathbb{N}$ and $\lambda_{j}, v_{j}, \gamma, q$, and $z \in \mathbb{C}(j=1, \ldots$, m) such that

$$
\sum_{j=1}^{m} \mathfrak{R}\left(\lambda_{j}\right)>\max \{0, \mathfrak{R}(q)-1\}, \quad \mathfrak{R}\left(v_{j}\right)>0, \mathfrak{R}(\gamma)>0, q \in(0,1) \cup \mathbb{N} .
$$

Also, let $|t|<1$. Then,

$$
\begin{aligned}
(1+t)^{-\sigma} E_{\left(\lambda_{j}, v_{j}\right)_{m}}^{\gamma, q}\left(\frac{z}{1+t}\right) & \\
& =\sum_{k=0}^{\infty}(-1)^{k}(\sigma)_{k} E_{\left(\lambda_{j}, v_{j}\right)_{m}, q}^{\infty, q} *{ }_{1} F_{1}(\sigma+k ; \sigma ;-z) \frac{t^{k}}{k !} .
\end{aligned}
$$

Corollary 2. Let $m \in \mathbb{N}$ and $\lambda_{j}, v_{j}, \gamma, q$, and $z \in \mathbb{C}(j=$ $1, \ldots, m)$ such that

$$
\sum_{j=1}^{m} \mathfrak{R}\left(\lambda_{j}\right)>\max \{0, \mathfrak{R}(q)-1\}, \quad \mathfrak{R}\left(v_{j}\right)>0, \mathfrak{R}(\gamma)>0, q \in(0,1) \cup \mathbb{N} .
$$

Also, let $|t|<1$. Then,

$$
\sum_{k=0}^{\infty}\left(\begin{array}{c}
\gamma+k-1 \\
k
\end{array}\right) E_{\left(\lambda_{j}, v_{j}\right)_{m}}^{\gamma+k, q}(z) t^{k}=(1-t)^{-\gamma} E_{\left(\lambda_{j}, v_{j}\right)_{m}}^{\gamma, q}\left(\frac{z}{(1-t)^{q}}\right) .
$$

The particular cases of (15), (23), (29), and (31) when $m=1$ give the following generating relations, stated, respectively, in Corollaries 3-6.

Corollary 3. Let $\sigma, \lambda, \nu, \gamma$, and $z \in \mathbb{C}$ such that $\mathfrak{R}(\lambda)>0$, $\mathfrak{R}(\nu) \geq-1, \mathfrak{R}(\gamma)>0$, and $q \in(0,1) \cup \mathbb{N}$. Also, let $|t|<1$. Then, 


$$
\begin{aligned}
(1 & +t)^{-\sigma} J_{\nu, q}^{\lambda, \gamma}\left(\frac{z}{1+t}\right) \\
& =\sum_{k=0}^{\infty}(-1)^{k}(\sigma)_{k} J_{\nu, q}^{\lambda, \gamma}(z) *{ }_{1} F_{1}(\sigma+k ; \sigma ;-z) \frac{t^{k}}{k !} .
\end{aligned}
$$

Corollary 4. Let $\sigma, \lambda, \nu, \gamma$, and $z \in \mathbb{C}$ such that $\mathfrak{R}(\lambda)>0$, $\mathfrak{R}(\nu) \geq-1, \mathfrak{R}(\gamma)>0$, and $q \in(0,1) \cup \mathbb{N}$. Also, let $|t|<1$. Then,

$$
\sum_{k=0}^{\infty}\left(\begin{array}{c}
\gamma+k-1 \\
k
\end{array}\right) J_{v, q}^{\lambda, \gamma+k}(z) t^{k}=(1-t)^{-\gamma} J_{v, q}^{\lambda, \gamma}\left(\frac{z}{(1-t)^{q}}\right) .
$$

Corollary 5. Let $\sigma, \lambda, v, \gamma$, and $z \in \mathbb{C}$ such that $\mathfrak{R}(\lambda)>0$, $\mathfrak{R}(\nu) \geq 0, \mathfrak{R}(\gamma)>0$, and $q \in(0,1) \cup \mathbb{N}$. Also, let $|t|<1$. Then,

$$
\begin{aligned}
(1+t)^{-\sigma} E_{\lambda, \nu}^{\gamma, q}\left(\frac{z}{1+t}\right) \\
\quad=\sum_{k=0}^{\infty}(-1)^{k}(\sigma)_{k} E_{\lambda, \nu}^{\gamma, q}(z) *{ }_{1} F_{1}(\sigma+k ; \sigma ;-z) \frac{t^{k}}{k !} .
\end{aligned}
$$

Corollary 6. Let $\sigma, \lambda, \nu, \gamma$, and $z \in \mathbb{C}$ such that $\Re(\lambda)>0$, $\mathfrak{R}(\nu) \geq 0, \mathfrak{R}(\gamma)>0$, and $q \in(0,1) \cup \mathbb{N}$. Also, let $|t|<1$. Then,

$$
\sum_{k=0}^{\infty}\left(\begin{array}{c}
\gamma+k-1 \\
k
\end{array}\right) E_{\lambda, \nu}^{\gamma+k, q}(z) t^{k}=(1-t)^{-\gamma} E_{\lambda, \nu}^{\gamma, q}\left(\frac{z}{(1-t)^{q}}\right) .
$$

\section{Data Availability}

No data were used to support this study.

\section{Conflicts of Interest}

The authors declare that there are no conflicts of interest regarding the publication of this paper.

\section{Acknowledgments}

This research has been partially supported by the Agencia Estatal de Investigacion (AEI) of Spain, co-financed by the European Fund for Regional Development (FEDER) corresponding to the 2014-2020 multiyear financial framework (project MTM2016-75140-P), and also supported by Xunta de Galicia (grant ED431C 2019/02). Shilpi Jain also thanks SERB (project number: MTR/2017/000194) for providing necessary facility.

\section{References}

[1] O. I. Marichev, Handbook of Integral Transforms and Higher Transcendental Functions, Ellis Horwood, Chichester, UK, 1983.
[2] R. S. Pathak, "Certain convergence theorems and asymptotic properties of a generalization of Lommel and Bessel-Maitland transform," Proceedings of the National Academy of Sciences, India, Section A, vol. 36, pp. 81-86, 1996.

[3] A. Wiman, "Über de fundamental satz in der theorie der funktionen $E_{\alpha}(x)$." Acta Mathematica, vol. 29, pp. 191-201, 1905.

[4] A. K. Shukla and J. C. Prajapati, "On a generalization of Mittag-Leffler function and its properties," Journal of Mathematical Analysis and Applications, vol. 336, no. 2, pp. 797-811, 2007.

[5] S. Jain and P. Agarwal, "A new class of integral relations involving general class of polynomials and I-functions," Walailak Journal of Science and Technology, vol. 12, no. 11, pp. 1009-1018, 2015.

[6] J. Choi and P. Agarwal, "A note on fractional integral operator associated with multiindex Mittag-Leffler functions," Filomat, vol. 30, no. 7, pp. 1931-1939, 2016.

[7] R. K. Saxena and K. Nishimoto, "N-fractional calculus of generalized Mittag-Leffler functions," Journal of Fractional Calculus, vol. 37, pp. 43-52, 2010.

[8] R. K. Saxena, T. K. Pogány, J. Ram, and J. Daiya, "Dirichlet averages of generalized multi-index Mittag-Leffler functions," Armenian Journal of Mathematics, vol. 3, no. 4, pp. 174-187, 2010.

[9] T. Pohlen, The Hadamard Product and Universal Power Series, Universität Trier, Trier, Germany, 2009.

[10] P. Agarwal and J. Choi, "Fractional calculus operators and their image formulas," Journal of the Korean Mathematical Society, vol. 53, no. 5, pp. 1183-1210, 2016.

[11] V. Kiryakova, "On two Saigos fractional integral operators in the class of univalent functions," Fractional Calculus and Applied Analysis, vol. 9, pp. 159-176, 2006.

[12] H. M. Srivastava, R. Agarwal, and S. Jain, "Integral transform and fractional derivative formulas involving the extended generalized hypergeometric functions and probability distributions," Mathematical Methods in the Applied Sciences, vol. 40, no. 1, pp. 255-273, 2017.

[13] R. Srivastava, R. Agarwal, and S. Jain, "A family of the incomplete hypergeometric functions and associated integral transform and fractional derivative formulas," Filomat, vol. 31, no. 1, pp. 125-140, 2017.

[14] P. Agarwal and C. L. Koul, "On generating functions," Journal of Rajasthan Academy of Physical Sciences, vol. 2, no. 3, pp. 173-180, 2003.

[15] P. Agarwal, M. Chand, and S. D. Purohit, "A note on generating functions involving the generalized Gauss hypergeometric functions," National Academy Science Letters, vol. 37, no. 5, pp. 457-459, 2014.

[16] P. Agarwal, M. Chand, and G. Singh, "On new sequence of functions involving the product of $F_{p}^{\alpha, \beta}(\cdot)$." Communications in Numerical Analysis, vol. 2015, no. 2, pp. 139-148, 2015.

[17] M. Chand, P. Agarwal, and J. Choi, "Note on generating relations associated with the generalized Gauss hypergeometric function," Applied Mathematical Sciences, vol. 10, no. 35, pp. 1747-1754, 2016.

[18] M.-P. Chen and H. M. Srivastava, "Orthogonality relations and generating functions for Jacobi polynomials and related hypergeometric functions," Applied Mathematics and Computation, vol. 68, no. 2-3, pp. 153-188, 1995.

[19] J. Choi and P. Agarwal, "Certain class of generating functions for the incomplete hypergeometric functions," Abstract and Applied Analysis, vol. 2014, Article ID 714560, 5 pages, 2014. 
[20] H. M. Srivastava, "Certain generating functions of several variables," ZAMM-Zeitschrift für Angewandte Mathematik und Mechanik, vol. 57, no. 6, pp. 339-340, 1977.

[21] H. M. Srivastava, "Generating relations and other results associated with some families of the extended Hurwitz-Lerch Zeta functions," SpringerPlus, vol. 2, no. 67, pp. 1-14, 2013.

[22] H. M. Srivastava, "A new family of the $\lambda$-generalized HurwitzLerch Zeta functions with applications," Applied Mathematics \& Information Sciences, vol. 8, no. 4, pp. 1485-1500, 2014.

[23] H. M. Srivastava and P. W. Karlsson, "Multiple Gaussian hypergeometric series," Ellis Horwood Series: Mathematics and Its Applications, Ellis Horwood Ltd., Chichester, UK, 1985.

[24] H. M. Srivastava and H. L. Manocha, A Treatise on Generating Functions, Ellis Horwood Ltd., Chichester, UK, 1984.

[25] H. M. Srivastava, M. A. Özarslan, and C. Kaanoğlu, "Some families of generating functions for a certain class of threevariable polynomials," Integral Transforms and Special Functions, vol. 21, no. 12, pp. 885-896, 2010.

[26] H. M. Srivastava and J. Choi, Zeta and q-Zeta Functions and Associated Series and Integrals, Elsevier Science Publishers, Amsterdam, Netherlands, 2012. 